\title{
Effect of Cytoplasm on Seed Yield and Attributing Traits in Sunflower (Helianthus annuus L.)
}

\author{
N.C. Sunitha* and Y.G. Shadakshari \\ University of Agricultural Sciences, Bengaluru- 560065, India \\ *Corresponding author
}

\begin{abstract}
A B S T R A C T
Keywords

Isonuclear alloplasmic,

Cytoplasm, CMS A

line, Maintainer line,

Combining ability, per

se performance

Article Info

Accepted:

04 January 2018

Available Online:

10 February 2018

The effect of cytoplasm on hybrid mean performance for seed yield and its contributing traits was investigated by evaluating 50 hybrids $(25 \mathrm{~A} \times \mathrm{R}$ and their corresponding $25 \mathrm{~B} \times \mathrm{R}$ hybrids) and 10 parents (five CMS A lines and corresponding maintainer lines) along with standard check hybrid DRSH-1. These isonuclear alloplasmic hybrids were observed for quantitative traits viz., days to 50 per cent flowering, head diameter, stem diameter, plant height, 100 seed weight, volume weight, days to maturity, hull content, seed yield plant ${ }^{-}$ ${ }^{1}$ and oil content. Significant variation was observed within the $A \times R$ and $B \times R$ group of hybrids for all the traits. But, differences between $A \times R$ and $B \times R$ group of hybrids were not significant for any trait except for hull content and oil content indicating poor influence of male sterility inducing cytoplasm. However, significant differences observed in few $\mathrm{A} \times \mathrm{R}$ and $\mathrm{B} \times \mathrm{R}$ hybrids for mean performance might be attributed to cytoplasm $\times$ nuclear interactions. The differences varied in their direction and magnitude without showing a definite pattern in favour of a particular cytoplasm. However, the differences so detected varied with the trait and nuclear genetic background.
\end{abstract}

\section{Introduction}

Cytoplasmic genes of plants including mitochondrial and chloroplast genes of plants are known to play a vital role in various metabolic processes. Apart from male sterility, cytoplasmic genes show influence on agronomic traits as reported in rice (Young and Virmani, 1990; Rosamma and Vijayakumar, 2007), chilli (Neelavva, 2012; Haritha, 2011), sorghum (Aruna et al., 2013; Sanjana et al., 2011; Reddy et al., 2009) and pearl millet (Chandrashekar et al., 2007; Yadav, 1999). Further, influence of cytoplasm on response to insects and diseases has been reported in pearl millet (Kumar, R. et al., 2010; Reddy et al., 2007). In commercial sunflower hybrid seed production, cytoplasmic male sterile (A) lines, sterility maintainer (B) lines and fertility restorer (R) lines are used. Elite Maintainer (B) lines which maintain sterility are backcrossed to sterile cytoplasm source for sufficient generations to obtain CMS A lines. Hence, CMS A lines and CMS B lines share same nuclear genes but differ for their cytoplasmic genes. Further, CMS A lines are maintained by crossing with maintainer lines and are crossed with restorer lines for Hybrid seed production. Since, the CMS B lines are used for the development and further maintenance 
of corresponding CMS A lines in sunflower breeding, the possible influence of cytoplasm on the key traits must be studied.

With the objective of determining the effect of cytoplasm on combining ability and yield attributes, we compared $\mathrm{A} \times \mathrm{R}$ hybrids with their corresponding $\mathrm{B} \times \mathrm{R}$ hybrids. Significant difference, if any, between them indicates the influence of cytoplasm on combining ability and yield attributes in sunflower. Studies have been reported in sesame (Mosjidis et al., 1984) and soybean (Davde et al., 1989) for cytoplasmic effects on oleic-linoleic fractions and protein content, respectively. Similar attempts have been made to study cytoplasmic effects in wheat (Liu and $\mathrm{Li}, 1994$ ), pearl millet (Thakur et al., 1991), rice (Kadoo et al., 2002), sorghum (Ramesh et al., 2006) and chilli (Neelavva, 2012). Cytoplasmic effect on seed yield, oil content and flowering time was reported by Serieys et al., (1992) by comparing reciprocal crosses. However, our present investigation was to determine cytoplasmic effect without evaluating reciprocal crosses but comparing alloplasmic isonuclear hybrids.

\section{Materials and Methods}

The material for the study comprised of five CMS lines viz., CMS 335A, CMS 17A, CMS 234A, CMS 135A and NDCMS 4A and their corresponding maintainer lines and five testers (RHA 6D-1, RHA 95-C-1, GKVK 3, RHA 93 and RHA 23). The five CMS lines (A-lines) were hand-pollinated using pollen collected from the five testers to produce $25 \mathrm{~A} \times \mathrm{R}$ crosses during kharif 2014. The maintainer counterpart of A-lines were induced male sterility by Gibberlic acid at 100 ppm during star bud stage and pollinated using the pollen collected from the same five testers to produce $25 \mathrm{~B} \times \mathrm{R}$ crosses during kharif 2014. A total of 50 hybrids $(25 \mathrm{~A} \times \mathrm{R}$ and $25 \mathrm{~B} \times \mathrm{R})$ were evaluated along with check (DRSH-1) and parents in contiguous blocks in randomized block design with two replications at Zonal Agricultural Research Station, UAS, GKVK, Bengaluru during Rabi 2014. All the recommended agronomic practices were followed to raise a good crop. Five competitive plants were taken randomly in each plot in each replication and observations were recorded on days to 50 per cent flowering, plant height $(\mathrm{cm})$, stem diameter $(\mathrm{cm})$, head diameter $(\mathrm{cm})$, stem diameter $(\mathrm{cm})$, Volume weight $(\mathrm{g} / 100 \mathrm{ml}), 100$ seed weight (g), Seed yield per plant (g), Hull content (\%), Oil content (\%) and Days to maturity. Replication-wise mean values were computed and used for statistical analysis.

Analysis of variance of $\mathrm{A} \times \mathrm{R}$ and $\mathrm{B} \times \mathrm{R}$ hybrids was carried out separately, the error mean sum of squares of individual analyses were pooled after testing their homogeneity (Bartlett, 1937). The pooled error mean sum of squares was used for estimating critical difference $(\mathrm{CD})$ to compare $\mathrm{A} \times \mathrm{R}$ and $\mathrm{B} \times \mathrm{R}$ hybrids in terms of the hybrid mean performance. The combining ability analysis was performed following Kempthorne (1957).

Since yield is associated with several other characters, positively with some and negatively with others, it is necessary to know the overall performance status of the parents / hybrids for all the characters simultaneously. The overall status of a parent or a cross with respect to per se performance was determined as per the method of Arunachalam and Bandopadhyay (1979) with slight modification as suggested by Mohan Rao (2001). The estimates of per se performance of hybrids were ranked by giving the highest rank for the parent or the cross which manifested the per se performance in desirable direction. The lowest rank was given for parent or the cross with the per se performance. This was repeated for each character except days to 50 per cent flowering, days to maturity and hull 
content $(\%)$ for which the ranking was given in reverse order. The ranks obtained by the parent / hybrid were summed up across all the characters to arrive at a total score for each of the parent / cross. Further, the mean of the total scores of all the genotypes (parents and hybrids) was computed which was used as the final norm to ascertain the status of a parent or a hybrid with respect to per se performance. The parent / hybrid whose total rank exceeded the final norm were given high $(\mathrm{H})$ overall per se performance. On the other hand, the parents or the cross, whose total rank was less than the final norm were given low (L) per se performance.

\section{Results and Discussion}

Both $\mathrm{A} \times \mathrm{R}$ and $\mathrm{B} \times \mathrm{R}$ groups of hybrids varied significantly among themselves as evident from the significant mean squares due to $\mathrm{A} \times \mathrm{R}$ and $\mathrm{B} \times \mathrm{R}$ hybrids. This variation so observed within the $\mathrm{A} \times \mathrm{R}$ and $\mathrm{B} \times \mathrm{R}$ hybrids provided statistical basis for comparing the $\mathrm{A}$ $\times \mathrm{R}$ and corresponding $\mathrm{B} \times \mathrm{R}$ hybrids to assess the effect of cytoplasm. However, $A \times R$ hybrids as a group differed significantly from $\mathrm{B} \times \mathrm{R}$ hybrids as a group only for hull content and oil content which is indicated by the significant mean squares due to $\mathrm{A} \times \mathrm{R}$ vs. $\mathrm{B} \times$ $\mathrm{R}$ hybrids as shown in table 1 .

\section{Cytoplasmic effects on combining ability}

Both A and B lines showed significant variation for all traits except head diameter, stem diameter, seed yield plant $^{-1}$ and hull content. While A lines varied significantly for head diameter while the B lines varied significantly for stem diameter and seed yield plant $^{-1}$ (Table 2). Mean squares due to both A lines $\times$ testers and $\mathrm{B}$ lines $\times$ testers were significant for plant height, seed yield plant $^{-1}$ and hull content. A linesxtesters were significant for head diameter, stem diameter and oil content. A $\times \mathrm{R}$ hybrids as a group differed significantly from the group of $B \times R$ hybrids only for hull content and oil content which is indicated by the significant mean squares due to $\mathrm{A} \times \mathrm{R}$ vs. $\mathrm{B} \times \mathrm{R}$ hybrids, indicating the possibility of presence of cytoplasmic influence.

\section{Cytoplasmic effects on per se performance of hybrids}

The mean performance of the $\mathrm{A} \times \mathrm{R}$ and corresponding $\mathrm{B} \times \mathrm{R}$ hybrids along with the difference between them is depicted in table 3 .

\section{Days to 50 per cent flowering}

Days to 50 per cent flowering ranged from 51 days (CMS 234A $\times$ RHA 23) to 69 days $(\mathrm{CMS} 335 \mathrm{~A} \times \mathrm{GKVK}-3)$ among $\mathrm{A} \times \mathrm{R}$ hybrids and from 52 days (CMS 234B $\times$ RHA 93) to 70 days $(\mathrm{CMS} 335 \mathrm{~B} \times \mathrm{GKVK}-3)$ in $\mathrm{B} \times \mathrm{R}$ hybrids. $\mathrm{A} \times \mathrm{R}$ and their corresponding $\mathrm{B} \times \mathrm{R}$ hybrids did not differ significantly for days to 50 per cent flowering with respect to their mean performance in any of the 25 nuclear genetic backgrounds. $\mathrm{A} \times \mathrm{R}$ group of hybrids and their corresponding $\mathrm{B} \times \mathrm{R}$ group of hybrids did not show significant difference for days to 50 per cent flowering which is evident from non-significant difference between overall means of $\mathrm{A} \times \mathrm{R}$ and $\mathrm{B} \times \mathrm{R}$ hybrids.

\section{Plant height}

Plant height ranged from $146.13 \mathrm{~cm}$ (NDCMS4A $\times$ RHA 93) to $188 \mathrm{~cm}$ (CMS 234A $\times$ RHA 95-C-1) among A $\times$ R hybrids . Among the $\mathrm{B} \times \mathrm{R}$ hybrids, it ranged from $142.5 \mathrm{~cm}$ (NDCMS4B $\times$ RHA 93) to $189.5 \mathrm{~cm}$ (CMS 335B $\times$ RHA 95-C-1). Significant differences between $\mathrm{A} \times \mathrm{R}$ and $\mathrm{B} \times \mathrm{R}$ crosses for per se performance were observed in three nuclear genetic backgrounds, of which two were in favour of male sterile cytoplasm and one was in favour of male fertile cytoplasm.

\section{Head diameter}


The variation among $\mathrm{A} \times \mathrm{R}$ hybrids and also $\mathrm{B}$ $\times$ Rhybrids was narrow for head diameter. Head diameter ranged from $12.25 \mathrm{~cm}$ (CMS $17 \mathrm{~A} \times \mathrm{RHA} 95-\mathrm{C}-1)$ to $17.03 \mathrm{~cm}($ NDCMS4A $\times$ GKVK-3) among the $\mathrm{A} \times \mathrm{R}$ hybrids while the range was $13.90 \mathrm{~cm}(\mathrm{CMS} 17 \mathrm{~B} \times \mathrm{RHA}$ 93) to $16 \mathrm{~cm}(\mathrm{CMS} 335 \mathrm{~B} \times \mathrm{RHA} 93)$ among the $\mathrm{B} \times \mathrm{R}$ hybrids.

Three of the 25 nuclear genetic backgrounds registered significant differences between $\mathrm{A} \times$ $\mathrm{R}$ and $\mathrm{B} \times \mathrm{R}$ hybrids for per se performance, of which, two were in favour of male sterile cytoplasm and one was in favour of male fertile cytoplasm.

\section{Stem diameter}

Among the $\mathrm{A} \times \mathrm{R}$ hybrids, stem diameter ranged from $1.97 \mathrm{~cm}(\mathrm{NDCMS} 4 \mathrm{~A} \times \mathrm{RHA} 23)$ to $3.10 \mathrm{~cm}(\mathrm{CMS} 17 \mathrm{~A} \times \mathrm{GKVK} 3)$ while it ranged from $1.77 \mathrm{~cm}$ (NDCMS4B $\times$ RHA 93) to $2.8 \mathrm{~cm}(\mathrm{CMS} 17 \mathrm{~A} \times \mathrm{RHA}$ 6D-1) among the $\mathrm{B} \times \mathrm{R}$ hybrids. $\mathrm{A} \times \mathrm{R}$ and $\mathrm{B} \times \mathrm{R}$ hybrids differed significantly for per se performance in two nuclear genetic backgrounds which were in favour of male sterile cytoplasm. The $\mathrm{A} \times \mathrm{R}$ group of hybrids and their corresponding $\mathrm{B} \times \mathrm{R}$ group of hybrids did not differ significantly for stem diameter which is evident from non-significant difference between overall means of $\mathrm{A} \times \mathrm{R}$ and $\mathrm{B} \times \mathrm{R}$ hybrids.

\section{Volume weight}

Volume weight ranged from $27 \mathrm{~g} / 100 \mathrm{ml}$ (CMS 17A $\times$ RHA 95-C-1) to $47.8 \mathrm{~g} / 100 \mathrm{ml}$ $(\mathrm{CMS}$ 135A $\times$ RHA 23) among $\mathrm{A} \times \mathrm{R}$ hybrids. Among $\mathrm{B} \times \mathrm{R}$ hybrids, it ranged from $26.05 \mathrm{~g} / 100 \mathrm{ml}(\mathrm{CMS} 17 \mathrm{~B} \times \mathrm{GKVK} 3)$ to $49.03 \mathrm{~g} / 100 \mathrm{ml}(\mathrm{CMS} 234 \mathrm{~B} \times \mathrm{RHA}$ 95-C-1). Three of the 25 nuclear genetic backgrounds registered significant differences for per se performance between $\mathrm{A} \times \mathrm{R}$ and $\mathrm{B} \times \mathrm{R}$ hybrids differing in their female cytoplasm, of which, two were in favour of male sterile cytoplasm and one was in favour of male fertile cytoplasm.

The $\mathrm{A} \times \mathrm{R}$ hybrids as a group did not show significant differences with their corresponding $\mathrm{B} \times \mathrm{R}$ group of hybrids for volume weight which is evident from nonsignificant difference between overall means of $\mathrm{A} \times \mathrm{R}$ and $\mathrm{B} \times \mathrm{R}$ hybrids.

\section{0 seed weight}

Among the $\mathrm{A} \times \mathrm{R}$ hybrids, 100 seed weight ranged from $3.33 \mathrm{~g}(\mathrm{CMS} 335 \mathrm{~A} \times \mathrm{RHA} 95-\mathrm{C}$ 1) to $7.24 \mathrm{~g}(\mathrm{NDCMS} 4 \mathrm{~A} \times \mathrm{RHA} 93)$ while it ranged from $3.23 \mathrm{~g}(335 \mathrm{~B} \times \mathrm{RHA} 95-\mathrm{C}-1)$ to $6.09 \mathrm{~g}(\mathrm{NDCMS} 4 \mathrm{~B} \times \mathrm{RHA} 23)$ among $\mathrm{B} \times \mathrm{R}$ hybrids. The per se performance of $A \times R$ and $\mathrm{B} \times \mathrm{R}$ hybrids were comparable and they differed only in three nuclear genetic backgrounds viz., CMS 335A/CMS335B × GKVK-3, CMS 17A/CMS 17B $\times$ RHA 95-C1 and NDCMS4A/NDCMS4B $\times$ RHA 95-C-1 for 100 seed weight. The male sterile cytoplasm based hybrids, CMS 17A $\times$ RHA 95-C-1, NDCMS4A $\times$ RHA 95-C-1 and a fertile cytoplasm based hybrid CMS335B $\times$ RHA 23 manifested high 100 seed weight. The $A \times R$ group of hybrids and their corresponding $\mathrm{B} \times \mathrm{R}$ group of hybrids were comparable for 100 seed weight which is evident from non-significant difference between overall means of $\mathrm{A} \times \mathrm{R}$ and $\mathrm{B} \times \mathrm{R}$ hybrids.

\section{Seed yield plant $^{-1}$}

Among the $\mathrm{A} \times \mathrm{R}$ hybrids, seed yield plant $^{-1}$ ranged from $39.9 \mathrm{~g}(\mathrm{NDCMS} 4 \mathrm{~A} \times \mathrm{RHA} 23)$ to $75.2 \mathrm{~g}(\mathrm{CMS} 135 \mathrm{~A} \times \mathrm{RHA} 95-\mathrm{C}-1)$ while it ranged from $28.5 \mathrm{~g}(\mathrm{NDCMS} 4 \mathrm{~A} \times \mathrm{RHA} 6 \mathrm{D}-$ 1) to $72.7 \mathrm{~g}(\mathrm{CMS} 135 \mathrm{~B} \times \mathrm{GKVK}-3)$ among $\mathrm{B}$ $\times$ R hybrids. 
Table.1 Combined analysis of variance of iso-nuclear alloplasmic hybrids for seed yield and its contributing traits in sunflower

\begin{tabular}{|c|c|c|c|c|c|c|}
\hline \multirow[t]{2}{*}{ Source of variation } & \multirow[t]{2}{*}{ df } & \multicolumn{5}{|c|}{ Mean sum of squares } \\
\hline & & $\begin{array}{l}\text { Days to } 50 \% \\
\text { flowering }\end{array}$ & $\begin{array}{c}\text { Plant } \\
\text { height } \\
(\mathrm{cm})\end{array}$ & $\begin{array}{c}\text { Head } \\
\text { diameter } \\
(\mathrm{cm})\end{array}$ & $\begin{array}{l}\text { Stem diameter } \\
(\mathrm{cm})\end{array}$ & $\begin{array}{c}\text { Volume weight } \\
(\mathrm{g} / 100 \mathrm{ml})\end{array}$ \\
\hline Replication & 1 & 1.96 & 87.98 & 0.12 & 0.02 & 22.08 \\
\hline Hybrids & 49 & $66.00 * *$ & $347.75 * *$ & $1.082 * *$ & $0.103 * *$ & $93.36 * *$ \\
\hline $\mathbf{A} \times \mathbf{R}$ & 24 & $73.19 * *$ & $329.81 * *$ & $1.69 * *$ & $0.114 * *$ & $99.14 * *$ \\
\hline $\mathbf{B} \times \mathbf{R}$ & 24 & $61.55^{* *}$ & $376.92 * *$ & 0.48 & $0.10 * *$ & $90.95 * *$ \\
\hline $\mathbf{A} \times \mathbf{R}$ vs. $\mathbf{B} \times \mathbf{R}$ & 1 & 0.04 & 78.32 & 0.81 & 0.01 & 12.41 \\
\hline Error & 49 & 5.82 & 28.21 & 0.47 & 0.03 & 11.69 \\
\hline
\end{tabular}

*Significant at $\mathrm{P}=0.05$, ** Significant at $\mathrm{P}=0.01$,

A: male sterile line, $\mathrm{B}$ : maintainer line, $\mathrm{R}$ : restorer line

Contd...

\begin{tabular}{|c|c|c|c|c|c|c|}
\hline \multirow{2}{*}{$\begin{array}{l}\text { Source of } \\
\text { variation }\end{array}$} & \multirow[t]{2}{*}{ df } & \multicolumn{5}{|c|}{ Mean sum of squares } \\
\hline & & $\begin{array}{c}100 \text { seed } \\
\text { weight }(\mathrm{g})\end{array}$ & $\begin{array}{l}\text { Seed yield } \\
\text { plant }^{-1}(\mathrm{~g})\end{array}$ & Days to maturity & $\begin{array}{c}\text { Hull content } \\
(\%)\end{array}$ & $\begin{array}{l}\text { Oil content } \\
(\%)\end{array}$ \\
\hline Replication & 1 & 0.89 & 0.12 & 0.09 & 5.26 & 0.85 \\
\hline Hybrids & 49 & $1.56 * *$ & $233.57 * *$ & $9.81 * *$ & $32.83 * *$ & $5.66^{* *}$ \\
\hline $\mathbf{A} \times \mathbf{R}$ & 24 & $1.88 * *$ & $239.27 * *$ & $11.50 * *$ & $23.74 * *$ & $6.36^{* *}$ \\
\hline $\mathbf{B} \times \mathbf{R}$ & 24 & $1.26 * *$ & $237.55^{* *}$ & $8.43 * *$ & $43.05 * *$ & $4.69 * *$ \\
\hline$A \times R$ vs. $B \times R$ & 1 & 1.16 & 1.06 & 2.25 & $5.82 * *$ & $12.39 * *$ \\
\hline Error & 49 & 0.55 & 21.76 & 1.27 & 3.37 & 1.28 \\
\hline
\end{tabular}

*Significant at $\mathrm{P}=0.05, * *$ Significant at $\mathrm{P}=0.01$

A: male sterile line, B: maintainer line, R: restorer line 
Table.2 Analysis of variance for combining ability of alloplasmic hybrids and their parents for seed yield and its Contributing traits in sunflower

\begin{tabular}{|c|c|c|c|c|c|c|c|c|c|c|c|}
\hline \multirow{3}{*}{$\begin{array}{l}\text { Source of } \\
\text { variation }\end{array}$} & \multirow[t]{3}{*}{ df } & \multicolumn{10}{|c|}{ Mean sum of squares } \\
\hline & & \multicolumn{2}{|c|}{$\begin{array}{l}\text { Days to } 50 \% \\
\text { flowering }\end{array}$} & \multicolumn{2}{|c|}{$\begin{array}{l}\text { Plant height } \\
\text { (cm) }\end{array}$} & \multicolumn{2}{|c|}{$\begin{array}{c}\text { Head } \\
\text { diameter } \\
(\mathrm{cm})\end{array}$} & \multicolumn{2}{|c|}{$\begin{array}{l}\text { Stem diameter } \\
(\mathrm{cm})\end{array}$} & \multicolumn{2}{|c|}{$\begin{array}{l}\text { Volume weight } \\
(\mathrm{g} / 100 \mathrm{ml})\end{array}$} \\
\hline & & $\mathbf{A} \times \mathbf{R}$ & $\mathbf{B} \times \mathbf{R}$ & $\mathbf{A} \times \mathbf{R}$ & $\mathbf{B} \times \mathbf{R}$ & $\mathbf{A} \times \mathbf{R}$ & $\mathbf{B} \times \mathbf{R}$ & $\mathbf{A} \times \mathbf{R}$ & $\mathbf{B} \times \mathbf{R}$ & $\mathbf{A} \times \mathbf{R}$ & $\mathbf{B} \times \mathbf{R}$ \\
\hline Replication & 1 & 0.72 & 1.28 & 70.09 & 23.94 & 0.003 & 0.19 & 0.02 & 0.001 & 19.82 & 3.19 \\
\hline Hybrids & 24 & $73.19 * *$ & $61.55^{* *}$ & $329.80 * *$ & $376.92 * *$ & $1.69 * *$ & 0.48 & $0.11 *$ & $0.09 * *$ & $96.26 * *$ & $90.95 * *$ \\
\hline Lines & 4 & $308.27 * *$ & $259.07 * *$ & $710.00 * *$ & $686.10 * *$ & $1.68 *$ & 0.72 & 0.11 & $0.28 * *$ & $438.39 * *$ & $386.02 * *$ \\
\hline Testers & 4 & $103.07 * *$ & $92.72 * *$ & $908.19 * *$ & $1022.64 * *$ & 0.61 & 0.29 & 0.10 & $0.14^{*}$ & 54.24 & 57.03 \\
\hline $\begin{array}{l}\text { Lines } x \\
\text { Testers } \\
\end{array}$ & 16 & 6.95 & 4.38 & $90.16 * *$ & $138.20 * *$ & $1.96^{* *}$ & 0.47 & $0.12 *$ & 0.04 & 21.23 & 25.66 \\
\hline Error & 24 & 3.59 & 8.28 & 19.74 & 37.59 & 0.39 & 0.56 & 0.04 & 0.02 & 10.57 & 13.98 \\
\hline
\end{tabular}

Significant at $\mathrm{P}=0.05, * *$ Significant at $\mathrm{P}=0.01$

Contd...

\begin{tabular}{|c|c|c|c|c|c|c|c|c|c|c|c|}
\hline \multirow{3}{*}{$\begin{array}{l}\text { Source of } \\
\text { variation }\end{array}$} & \multirow[t]{3}{*}{ Df } & \multicolumn{10}{|c|}{ Mean sum of squares } \\
\hline & & \multicolumn{2}{|c|}{$\begin{array}{c}100 \text { seed } \\
\text { weight }(\mathrm{g})\end{array}$} & \multicolumn{2}{|c|}{$\begin{array}{l}\text { Seed yield plant } \\
\text { (g) }\end{array}$} & \multicolumn{2}{|c|}{ Days to maturity } & \multicolumn{2}{|c|}{$\begin{array}{l}\text { Hull content } \\
(\%)\end{array}$} & \multicolumn{2}{|c|}{$\begin{array}{l}\text { Oil content } \\
(\%)\end{array}$} \\
\hline & & $\mathbf{A} \times \mathbf{R}$ & $\mathbf{B} \times \mathbf{R}$ & $\mathbf{A} \times \mathbf{R}$ & $\mathbf{B} \times \mathbf{R}$ & $\mathbf{A} \times \mathbf{R}$ & $\mathbf{B} \times \mathbf{R}$ & $\mathbf{A} \times \mathbf{R}$ & $\mathbf{B} \times \mathbf{R}$ & $\mathbf{A} \times \mathbf{R}$ & $\mathbf{B} \times \mathbf{R}$ \\
\hline Replication & 1 & 1.11 & 0.07 & 0.387 & 1.15 & 0.08 & 0.50 & 42.55 & 5.38 & 1.94 & 0.008 \\
\hline Hybrids & 24 & $1.88 * *$ & 1.25 & $239.08 * *$ & $237.55^{* *}$ & $11.50 * *$ & $8.44 * *$ & $148.02 * *$ & $287.45^{* *}$ & $6.36^{* *}$ & $4.69 *$ \\
\hline Lines & 4 & $8.90^{* *}$ & $3.11 * *$ & 332.04 & $544.20^{*}$ & $52.92 * *$ & $38.57 * *$ & 235.91 & 395.71 & $17.47 * *$ & $14.89 * *$ \\
\hline Testers & 4 & 0.10 & $2.90 * *$ & 453.34 & 256.26 & $9.77 * *$ & $5.27 *$ & 247.92 & 523.03 & 8.55 & $6.78 *$ \\
\hline $\begin{array}{l}\text { Lines } x \\
\text { Testers }\end{array}$ & 16 & 0.57 & 0.38 & $162.27 * *$ & $156.22 * *$ & 1.58 & 1.69 & $101.07 * *$ & 201.50** & $3.03 * *$ & 1.62 \\
\hline Error & 24 & 0.46 & 0.63 & 17.31 & 27.01 & 0.99 & 1.58 & 26.64 & 21.90 & 0.73 & 1.85 \\
\hline
\end{tabular}

*Significant at $\mathrm{P}=0.05, * *$ Significant at $\mathrm{P}=0.01$ 
Table.3 Comparison of per se performance of $\mathrm{A} \times \mathrm{R}$ and $\mathrm{B} \times \mathrm{R}$ sunflower hybrids for seed yield and its attributing traits

\begin{tabular}{|c|c|c|c|c|c|c|c|c|c|c|c|c|}
\hline \multirow[t]{2}{*}{ Nuclear genetic background } & \multicolumn{3}{|c|}{ Days to $50 \%$ flowering } & \multicolumn{3}{|c|}{ Plant height (cm) } & \multicolumn{3}{|c|}{ Stem diameter $(\mathbf{c m})$} & \multicolumn{3}{|c|}{ Head diameter (cm) } \\
\hline & $\mathbf{A} \times \mathbf{R}$ & $\mathbf{B} \times \mathbf{R}$ & Diff. & $\mathbf{A} \times \mathbf{R}$ & $\mathbf{B} \times \mathbf{R}$ & Diff. & $\mathbf{A} \times \mathbf{R}$ & $\mathbf{B} \times \mathbf{R}$ & Diff. & $\mathbf{A} \times \mathbf{R}$ & $\mathbf{B} \times \mathbf{R}$ & Diff. \\
\hline CMS 335A/CMS335B $\times$ RHA 6D-1 & 69.0 & 68.5 & 0.50 & 176.50 & 168.40 & 8.10 & 2.67 & 2.54 & 0.13 & 15.90 & 15.30 & 0.60 \\
\hline CMS 335A/CMS335B $\times$ RHA 95-C-1 & 68.0 & 69.0 & -1.00 & 187.00 & 189.50 & -2.50 & 2.47 & 2.57 & -0.10 & 15.40 & 15.40 & 0.00 \\
\hline CMS 335A/CMS335B $\times$ GKVK-3 & 69.5 & 70.5 & -1.00 & 187.00 & 180.30 & 6.70 & 2.21 & 2.56 & $-0.35 *$ & 14.50 & 15.20 & -0.70 \\
\hline CMS 335A/CMS335B $\times$ RHA 93 & 64.5 & 65.5 & -1.00 & 173.10 & 171.10 & 2.00 & 2.61 & 2.46 & 0.15 & 16.10 & 16.00 & 0.10 \\
\hline CMS 335A/CMS335B $\times$ RHA 23 & 65.0 & 66.5 & -1.50 & 170.54 & 182.50 & $-11.96 *$ & 2.58 & 2.62 & -0.04 & 15.50 & 15.10 & 0.40 \\
\hline CMS 17A/CMS 17B $\times$ RHA 6D-1 & 63.0 & 63.0 & 0.00 & 174.38 & 171.50 & 2.88 & 2.51 & 2.80 & -0.29 & 15.50 & 15.40 & 0.10 \\
\hline CMS 17A/CMS 17B $\times$ RHA 95-C-1 & 68.5 & 64.0 & 4.50 & 172.50 & 176.20 & -3.70 & 2.00 & 2.72 & -0.72 & 12.25 & 15.10 & $-2.85 * *$ \\
\hline CMS 17A/CMS 17B $\times$ GKVK-3 & 65.0 & 66.0 & -1.00 & 186.80 & 180.80 & 6.00 & 3.10 & 2.50 & 0.60 & 16.50 & 14.90 & $1.60^{*}$ \\
\hline CMS 17A/CMS 17B $\times$ RHA 93 & 55.0 & 57.0 & -2.00 & 154.75 & 159.80 & -5.05 & 2.47 & 2.50 & -0.03 & 14.60 & 13.90 & 0.70 \\
\hline CMS 17A/CMS 17B $\times$ RHA 23 & 59.0 & 58.5 & 0.50 & 169.25 & 160.80 & 8.45 & 2.67 & 2.50 & 0.17 & 15.83 & 15.20 & 0.63 \\
\hline CMS 234A/CMS 234B $\times$ RHA 6D-1 & 53.0 & 54.0 & -1.00 & 170.00 & 154.50 & $15.50 *$ & 2.50 & 2.29 & 0.21 & 15.10 & 15.30 & -0.20 \\
\hline CMS 234A/CMS 234B $\times$ RHA 95-C-1 & 56.5 & 58.0 & -1.50 & 188.00 & 182.50 & 5.50 & 2.47 & 2.42 & 0.05 & 16.20 & 15.50 & 0.70 \\
\hline CMS 234A/CMS 234B× GKVK-3 & 59.0 & 60.5 & -1.50 & 185.50 & 181.30 & 4.20 & 2.46 & 2.56 & -0.10 & 14.90 & 15.90 & -1.00 \\
\hline CMS 234A/CMS 234B× RHA 93 & 52.5 & 52.5 & 0.00 & 163.00 & 155.30 & 7.70 & 2.22 & 2.16 & 0.06 & 14.50 & 15.30 & -0.80 \\
\hline CMS 234A/CMS 234B $\times$ RHA 23 & 51.0 & 53.5 & -2.50 & 156.50 & 158.50 & -2.00 & 2.23 & 2.15 & 0.08 & 14.90 & 14.90 & 0.00 \\
\hline CMS 135A/CMS 135B $\times$ RHA 6D-1 & 55.5 & 58.0 & -2.50 & 152.50 & 158.40 & -5.90 & 2.20 & 2.23 & -0.03 & 14.90 & 14.50 & 0.40 \\
\hline CMS 135A/CMS 135B $\times$ RHA 95-C-1 & 58.0 & 59.0 & -1.00 & 177.00 & 174.00 & 3.00 & 2.28 & 2.41 & -0.13 & 15.70 & 15.80 & -0.10 \\
\hline CMS 135A/CMS 135B× GKVK-3 & 60.5 & 61.0 & -0.50 & 162.00 & 173.50 & -11.50 & 2.44 & 2.51 & -0.07 & 15.20 & 15.10 & 0.10 \\
\hline CMS 135A/CMS 135B $\times$ RHA 93 & 52.5 & 56.0 & -3.50 & 146.50 & 146.10 & 0.40 & 2.26 & 2.21 & 0.05 & 15.10 & 15.00 & 0.10 \\
\hline CMS 135A/CMS 135B $\times$ RHA 23 & 52.0 & 53.0 & -1.00 & 154.10 & 157.60 & -3.50 & 2.26 & 2.46 & -0.20 & 14.80 & 15.80 & -1.00 \\
\hline NDCMS4A/NDCMS4B $\times$ RHA 6D-1 & 54.5 & 56.0 & -1.50 & 164.00 & 164.00 & 0.00 & 2.46 & 2.33 & 0.13 & 16.43 & 14.97 & 1.47 \\
\hline NDCMS4A/NDCMS4B $\times$ RHA 95-C-1 & 55.5 & 55.5 & 0.00 & 163.00 & 150.00 & $13.00 *$ & 2.47 & 2.28 & 0.19 & 15.80 & 14.70 & 1.10 \\
\hline NDCMS4A/NDCMS4B× GK VK-3 & 60.0 & 63.5 & -3.50 & 173.25 & 180.00 & -6.75 & 2.69 & 2.49 & 0.20 & 17.03 & 15.00 & $2.03 *$ \\
\hline NDCMS4A/NDCMS4B× RHA 93 & 52.0 & 53.5 & -1.50 & 146.13 & 142.50 & 3.63 & 2.33 & 1.77 & $0.5^{* *}$ & 15.63 & 14.20 & 1.43 \\
\hline NDCMS4A/NDCMS4B $\times$ RHA 23 & 54.0 & 54.5 & -0.50 & 151.50 & 149.50 & 2.00 & 1.97 & 2.11 & -0.14 & 15.00 & 15.30 & -0.30 \\
\hline Overall mean & 58.9 & 59.8 & -0.90 & 168.19 & 166.74 & 1.45 & 2.42 & 2.41 & 0.01 & 15.33 & 15.15 & 0.18 \\
\hline Sem. \pm & 1.39 & 1.98 & 1.72 & 3.82 & 5.09 & 4.50 & 0.13 & 0.09 & 0.11 & 0.51 & 0.57 & 0.543 \\
\hline $\mathrm{CD} @ \mathrm{P}=0.05^{*}$ & 4.02 & 5.71 & 4.94 & 11 & 14.63 & 11.94 & 0.39 & 0.27 & 0.33 & 1.46 & 1.65 & 1.56 \\
\hline$C D @ P=0.01^{* * *}$ & 5.39 & 7.67 & 6.63 & 14.77 & 19.65 & 17.38 & 0.52 & 0.37 & 0.45 & 1.96 & 2.22 & 2.09 \\
\hline
\end{tabular}

A: male sterile line, B: maintainer line, $\mathrm{R}$ : restorer line, *Significant at $\mathrm{P}=0.05$, ** Significant at $\mathrm{P}=0.01$ 
Contd...

\begin{tabular}{|c|c|c|c|c|c|c|c|c|c|}
\hline \multirow[t]{2}{*}{ Nuclear genetic background } & \multicolumn{3}{|c|}{ Volume weight (g/100ml) } & \multicolumn{3}{|c|}{100 seed weight (g) } & \multicolumn{3}{|c|}{ Hull content (\%) } \\
\hline & $\mathbf{A} \times \mathbf{R}$ & $\mathbf{B} \times \mathbf{R}$ & Diff. & $\mathbf{A} \times \mathbf{R}$ & $\mathbf{B} \times \mathbf{R}$ & Diff. & $\mathbf{A} \times \mathbf{R}$ & $\mathbf{B} \times \mathbf{R}$ & Diff. \\
\hline CMS 335A/CMS335B $\times$ RHA 6D-1 & 28.67 & 27.40 & 1.27 & 4.11 & 5.05 & -0.94 & 31.30 & 32.69 & 1.39 \\
\hline CMS 335A/CMS335B $\times$ RHA 95-C-1 & 31.90 & 39.20 & -7.30 & 4.81 & 4.58 & 0.23 & 28.32 & 32.12 & $-3.80 *$ \\
\hline CMS 335A/CMS335B $\times$ GKVK-3 & 28.81 & 32.15 & -3.34 & 3.51 & 5.67 & $-2.16^{* *}$ & 32.44 & 35.72 & -3.28 \\
\hline CMS 335A/CMS335B $\times$ RHA 93 & 27.06 & 29.76 & -2.70 & 3.33 & 3.23 & 0.10 & 34.99 & 31.77 & 3.22 \\
\hline CMS 335A/CMS335B $\times$ RHA 23 & 27.90 & 26.05 & 1.85 & 4.90 & 4.38 & 0.52 & 33.98 & 32.51 & 1.47 \\
\hline CMS 17A/CMS 17B $\times$ RHA 6D-1 & 36.57 & 41.49 & -4.92 & 4.47 & 4.11 & 0.36 & 33.27 & 33.51 & -0.24 \\
\hline CMS 17A/CMS 17B $\times$ RHA 95-C-1 & 27.00 & 33.99 & -6.99 & 5.36 & 3.29 & $2.07 * *$ & 35.95 & 44.53 & $-8.58 * *$ \\
\hline CMS 17A/CMS 17Bx GKVK-3 & 36.00 & 39.44 & -3.44 & 5.00 & 4.05 & 0.96 & 38.88 & 43.39 & $-4.51^{*}$ \\
\hline CMS 17A/CMS 17B $\times$ RHA 93 & 47.18 & 46.42 & 0.77 & 4.14 & 4.08 & 0.07 & 34.82 & 36.58 & -1.77 \\
\hline CMS 17A/CMS 17B $\times$ RHA 23 & 40.48 & 41.72 & -1.25 & 4.92 & 5.15 & -0.23 & 41.35 & 35.58 & $5.77 * *$ \\
\hline CMS 234A/CMS 234B $\times$ RHA 6D-1 & 45.31 & 42.66 & 2.65 & 5.38 & 5.29 & 0.09 & 30.92 & 28.68 & 2.24 \\
\hline CMS 234A/CMS 234B $\times$ RHA 95-C-1 & 41.79 & 49.03 & $-7.24 *$ & 4.51 & 5.26 & -0.76 & 36.81 & 42.62 & $-5.81 * *$ \\
\hline CMS 234A/CMS 234B $\times$ GKVK-3 & 43.34 & 40.02 & 3.32 & 4.48 & 5.42 & -0.94 & 28.69 & 33.47 & $-4.78 *$ \\
\hline CMS 234A/CMS 234B $\times$ RHA 93 & 44.04 & 45.36 & -1.33 & 4.55 & 4.38 & 0.17 & 35.32 & 33.19 & 2.13 \\
\hline CMS 234A/CMS 234B $\times$ RHA 23 & 44.53 & 45.24 & -0.72 & 5.15 & 6.00 & -0.85 & 32.32 & 28.01 & $4.31 *$ \\
\hline CMS 135A/CMS 135B $\times$ RHA 6D-1 & 44.62 & 39.21 & 5.41 & 4.90 & 5.09 & -0.20 & 30.25 & 43.50 & $-13.2 * *$ \\
\hline CMS 135A/CMS 135B $\times$ RHA 95-C-1 & 40.74 & 43.24 & -2.50 & 5.29 & 4.73 & 0.57 & 41.42 & 39.20 & 2.22 \\
\hline CMS 135A/CMS 135B $\times$ GKVK-3 & 44.28 & 45.73 & -1.45 & 4.93 & 4.91 & 0.02 & 33.86 & 33.28 & 0.58 \\
\hline CMS 135A/CMS 135B $\times$ RHA 93 & 44.51 & 42.25 & 2.26 & 5.26 & 4.84 & 0.42 & 33.44 & 32.29 & 1.15 \\
\hline CMS 135A/CMS 135B $\times$ RHA 23 & 47.80 & 46.34 & 1.46 & 5.72 & 5.40 & 0.32 & 35.30 & 34.97 & 0.32 \\
\hline NDCMS4A/NDCMS4B $\times$ RHA 6D-1 & 45.69 & 35.68 & $10.1^{* *}$ & 6.12 & 5.41 & 0.71 & 33.09 & 37.12 & $-4.04 *$ \\
\hline NDCMS4A/NDCMS4B $\times$ RHA 95-C-1 & 45.40 & 30.46 & $14.9 * *$ & 6.66 & 4.30 & $2.37 * *$ & 34.50 & 34.99 & -0.49 \\
\hline NDCMS4A/NDCMS4B $\times$ GK VK-3 & 40.30 & 35.38 & 4.92 & 6.53 & 6.07 & 0.46 & 31.87 & 30.55 & 1.32 \\
\hline NDCMS4A/NDCMS4B $\times$ RHA 93 & 45.45 & 43.52 & 1.93 & 7.24 & 5.83 & 1.41 & 32.06 & 31.61 & 0.45 \\
\hline NDCMS4A/NDCMS4B $\times$ RHA 23 & 44.24 & 44.34 & -0.10 & 6.75 & 6.09 & 0.65 & 35.13 & 32.64 & 2.49 \\
\hline Overall mean & 39.74 & 39.04 & 0.70 & 5.12 & 4.90 & 0.22 & 34.98 & 35.46 & -0.48 \\
\hline Sem. \pm & 2.22 & 2.74 & 2.49 & 0.49 & 0.51 & 0.50 & 1.29 & 1.25 & 1.27 \\
\hline $\mathrm{CD} @ \mathrm{P}=0.05^{*}$ & 6.39 & 7.88 & 7.17 & 1.41 & 1.46 & 1.43 & 3.70 & 3.60 & 3.65 \\
\hline $\mathrm{CD} @ \mathrm{P}=0.01^{* * *}$ & 8.58 & 10.58 & 9.63 & 1.89 & 1.96 & 1.93 & 4.96 & 4.83 & 4.90 \\
\hline
\end{tabular}

A: male sterile line, B: maintainer line, $\mathrm{R}$ : restorer line, *Significant at $\mathrm{P}=0.05, * *$ Significant at $\mathrm{P}=0.01$ 
Contd...

\begin{tabular}{|c|c|c|c|c|c|c|c|c|c|}
\hline \multirow[t]{2}{*}{ Nuclear genetic background } & \multicolumn{3}{|c|}{ Days to maturity } & \multicolumn{3}{|c|}{ Seed yield per plant $(\mathrm{g})$} & \multicolumn{3}{|c|}{ Oil content (\%) } \\
\hline & $\mathbf{A} \times \mathbf{R}$ & $\mathbf{B} \times \mathbf{R}$ & Diff. & $\mathbf{A} \times \mathbf{R}$ & $\mathbf{B} \times \mathbf{R}$ & Diff. & $\mathbf{A} \times \mathbf{R}$ & $\mathbf{B} \times \mathbf{R}$ & Diff. \\
\hline CMS 335A/CMS335B $\times$ RHA 6D-1 & 97.50 & 96.00 & 1.50 & 45.20 & 48.30 & -3.10 & 36.79 & 36.37 & 0.42 \\
\hline CMS 335A/CMS335B $\times$ RHA 95-C-1 & 97.00 & 97.00 & 0.00 & 48.60 & 57.20 & -8.60 & 34.30 & 36.79 & $-2.49 *$ \\
\hline CMS 335A/CMS335B $\times$ GKVK-3 & 97.00 & 98.50 & -1.50 & 48.30 & 46.40 & 1.90 & 35.89 & 38.39 & $-2.50 *$ \\
\hline CMS 335A/CMS335B $\times$ RHA 93 & 96.50 & 93.50 & $3.00 * *$ & 43.80 & 45.00 & -1.20 & 33.15 & 36.45 & $-3.30 * *$ \\
\hline CMS 335A/CMS335B $\times$ RHA 23 & 97.50 & 97.00 & 0.50 & 47.50 & 51.40 & -3.90 & 36.51 & 36.21 & 0.31 \\
\hline CMS 17A/CMS 17B $\times$ RHA 6D-1 & 92.00 & 91.00 & 1.00 & 47.50 & 59.30 & $-11.80^{*}$ & 35.56 & 33.81 & 1.76 \\
\hline CMS 17A/CMS 17B $\times$ RHA 95-C-1 & 92.50 & 92.00 & 0.50 & 46.00 & 39.80 & 6.20 & 34.13 & 33.72 & 0.41 \\
\hline CMS 17A/CMS 17B $\times$ GKVK-3 & 96.50 & 93.50 & $3.00 * *$ & 71.00 & 65.20 & 5.80 & 33.47 & 36.54 & $-3.07 * *$ \\
\hline CMS 17A/CMS 17B $\times$ RHA 93 & 94.00 & 93.00 & 1.00 & 70.30 & 69.70 & 0.60 & 35.84 & 35.30 & 0.55 \\
\hline CMS 17A/CMS 17B $\times$ RHA 23 & 93.50 & 93.00 & 0.50 & 42.40 & 55.10 & $-12.70 * *$ & 33.59 & 33.63 & -0.04 \\
\hline CMS 234A/CMS 234B $\times$ RHA 6D-1 & 91.50 & 92.00 & -0.50 & 55.90 & 44.50 & $11.40^{*}$ & 38.94 & 38.29 & 0.66 \\
\hline CMS 234A/CMS 234B $\times$ RHA 95-C-1 & 92.00 & 92.50 & -0.50 & 62.90 & 63.10 & -0.20 & 38.13 & 37.92 & 0.20 \\
\hline CMS 234A/CMS 234B $\times$ GK VK-3 & 94.00 & 94.00 & 0.00 & 64.20 & 72.70 & -8.50 & 36.30 & 36.61 & -0.31 \\
\hline CMS 234A/CMS 234B $\times$ RHA 93 & 90.50 & & & 60.40 & 53.90 & 6.50 & 37.98 & 38.57 & -0.59 \\
\hline CMS 234A/CMS 234B $\times$ RHA 23 & 91.50 & 92.00 & -0.50 & 47.80 & 47.40 & 0.40 & 34.78 & 36.59 & -1.81 \\
\hline CMS 135A/CMS 135B $\times$ RHA 6D-1 & 92.00 & 90.50 & 1.50 & 45.15 & 49.60 & -4.45 & 38.15 & 37.73 & 0.42 \\
\hline CMS 135A/CMS 135B $\times$ RHA 95-C-1 & 93.00 & 92.50 & 0.50 & 75.20 & 70.00 & 5.20 & 38.17 & 38.24 & -0.06 \\
\hline CMS 135A/CMS 135B $\times$ GKVK-3 & 94.00 & 92.50 & 1.50 & 64.00 & 51.60 & $12.40 * *$ & 36.59 & 38.01 & -1.43 \\
\hline CMS 135A/CMS 135B $\times$ RHA 93 & 91.50 & 92.50 & -1.00 & 42.70 & 53.30 & $-10.60 *$ & 38.73 & 37.55 & 1.19 \\
\hline CMS 135A/CMS 135B $\times$ RHA 23 & 91.00 & 91.50 & -0.50 & 40.50 & 55.00 & $-14.50 * *$ & 35.66 & 36.23 & -0.57 \\
\hline NDCMS4A/NDCMS4B $\times$ RHA 6D-1 & 91.00 & 91.50 & -0.50 & 42.20 & 28.50 & $13.70 * *$ & 37.69 & 36.38 & 1.32 \\
\hline NDCMS4A/NDCMS4B $\times$ RHA 95-C-1 & 91.00 & 91.50 & -0.50 & 41.90 & 40.70 & 1.20 & 35.14 & 36.54 & -1.40 \\
\hline NDCMS4A/NDCMS4B $\times$ GKVK-3 & 93.50 & 92.00 & 1.50 & 59.90 & 54.00 & 5.90 & 34.42 & 39.13 & -4.71 \\
\hline NDCMS4A/NDCMS4B $\times$ RHA 93 & 90.00 & 91.00 & -1.00 & 40.00 & 38.10 & 1.90 & 33.87 & 36.46 & $-2.60 *$ \\
\hline NDCMS4A/NDCMS4B $\times$ RHA 23 & 91.50 & 91.50 & 0.00 & 39.90 & 38.60 & 1.30 & 34.59 & 34.53 & 0.06 \\
\hline Overall mean & 93.28 & 92.98 & 0.30 & 51.73 & 51.94 & -0.21 & 35.93 & 36.64 & -0.71 \\
\hline Sem. \pm & 0.71 & 0.83 & 0.78 & 2.97 & 3.15 & 3.06 & 0.56 & 0.82 & 0.70 \\
\hline$C D @ P=0.05^{*}$ & 2.05 & 2.40 & 2.23 & 8.52 & 9.06 & 8.80 & 1.60 & 2.36 & 2.01 \\
\hline$C D @ P=0.01 * *$ & 2.75 & 3.22 & 2.99 & 11.44 & 12.17 & 11.81 & 2.15 & 3.16 & 2.70 \\
\hline
\end{tabular}

A: male sterile line, B: maintainer line, $\mathrm{R}$ : restorer line, *Significant at $\mathrm{P}=0.05, * *$ Significant at $\mathrm{P}=0.01$ 
Table.4 Overall status of hybrids across traits for per se performance

\begin{tabular}{|c|c|c|c|c|}
\hline \multirow[t]{2}{*}{ Nuclear genetic background } & \multicolumn{2}{|c|}{ Rank } & \multicolumn{2}{|c|}{ Overall per se performance } \\
\hline & $\mathbf{A} \times \mathbf{R}$ & $\mathbf{B} \times \mathbf{R}$ & $\mathbf{A} \times \mathbf{R}$ & $\mathbf{B} \times \mathbf{R}$ \\
\hline CMS 335A/CMS335B $\times$ RHA 6D-1 & 147 & 156 & $\mathrm{H}$ & $\mathrm{H}$ \\
\hline CMS 335A/CMS335B $\times$ RHA 95-C-1 & 177 & 156 & $\mathrm{H}$ & $\mathrm{H}$ \\
\hline CMS 335A/CMS335B× GKVK-3 & 156 & 125 & $\mathrm{H}$ & $\mathrm{L}$ \\
\hline CMS 335A/CMS335B $\times$ RHA 93 & 132 & 133 & $\mathrm{~L}$ & $\mathrm{~L}$ \\
\hline CMS 335A/CMS335B $\times$ RHA 23 & 140 & 145 & $\mathrm{~L}$ & $\mathrm{H}$ \\
\hline CMS 17A/CMS 17B $\times$ RHA 6D-1 & 141 & 110 & $\mathrm{~L}$ & $\mathrm{~L}$ \\
\hline CMS 17A/CMS 17B $\times$ RHA 95-C-1 & 157 & 163 & $\mathrm{H}$ & $\mathrm{H}$ \\
\hline CMS 17A/CMS 17B $\times$ GKVK-3 & 83 & 100 & $\mathrm{~L}$ & $\mathrm{~L}$ \\
\hline CMS 17A/CMS 17B $\times$ RHA 93 & 119 & 119 & $\mathrm{~L}$ & $\mathrm{~L}$ \\
\hline CMS 17A/CMS 17B $\times$ RHA 23 & 152 & 128 & $\mathrm{H}$ & $\mathrm{L}$ \\
\hline CMS 234A/CMS 234B $\times$ RHA 6D-1 & 114 & 170 & $\mathrm{~L}$ & $\mathrm{H}$ \\
\hline CMS 234A/CMS 234B $\times$ RHA 95-C-1 & 87 & 73 & $\mathrm{~L}$ & $\mathrm{~L}$ \\
\hline CMS 234A/CMS 234B $\times$ GK VK-3 & 125 & 77 & $\mathrm{~L}$ & $\mathrm{~L}$ \\
\hline CMS 234A/CMS 234B $\times$ RHA 93 & 130 & 143 & $\mathrm{~L}$ & $\mathrm{H}$ \\
\hline CMS 234A/CMS 234B $\times$ RHA 23 & 158 & 163 & $\mathrm{H}$ & $\mathrm{H}$ \\
\hline CMS 135A/CMS 135B $\times$ RHA 6D-1 & 166 & 143 & $\mathrm{H}$ & $\mathrm{H}$ \\
\hline CMS 135A/CMS 135B $\times$ RHA 95-C-1 & 73 & 81 & $\mathrm{~L}$ & $\mathrm{~L}$ \\
\hline CMS 135A/CMS 135B $\times$ GK VK-3 & 113 & 117 & $\mathrm{~L}$ & $\mathrm{~L}$ \\
\hline CMS 135A/CMS 135B× RHA 93 & 249 & 155 & $\mathrm{H}$ & $\mathrm{H}$ \\
\hline CMS 135A/CMS 135B× RHA 23 & 160 & 123 & $\mathrm{H}$ & $\mathrm{L}$ \\
\hline NDCMS4A/NDCMS4B $\times$ RHA 6D-1 & 134 & 167 & $\mathrm{~L}$ & $\mathrm{H}$ \\
\hline NDCMS4A/NDCMS4B $\times$ RHA 95-C-1 & 133 & 195 & $\mathrm{~L}$ & $\mathrm{H}$ \\
\hline NDCMS4A/NDCMS4B $\times$ GKVK-3 & 110 & 110 & $\mathrm{~L}$ & $\mathrm{~L}$ \\
\hline NDCMS4A/NDCMS4B $\times$ RHA 93 & 177 & 199 & $\mathrm{H}$ & $\mathrm{H}$ \\
\hline NDCMS4A/NDCMS4B $\times$ RHA 23 & 164 & 179 & $\mathrm{H}$ & $\mathrm{H}$ \\
\hline Overall mean & 147 & 156 & $\mathrm{H}$ & $\mathrm{H}$ \\
\hline Sem. \pm & 177 & 156 & $\mathrm{H}$ & $\mathrm{H}$ \\
\hline CD @ P $=0.05 *$ & 156 & 125 & $\mathrm{H}$ & $\mathrm{L}$ \\
\hline$C D @ P=0.01 * *$ & 132 & 133 & $\mathrm{~L}$ & $\mathrm{~L}$ \\
\hline
\end{tabular}

Final norm= 143. L- Low overall specific hybrid combination, H-high overall specific hybrid combination, A: male sterile line, B: maintainer line, R: restorer line

Significant differences in per se performance for seed yield plant $^{-1}$ were observed between $\mathrm{A} \times \mathrm{R}$ and $\mathrm{B} \times \mathrm{R}$ hybrids in seven nuclear genetic backgrounds, of which, three viz., CMS 234A/CMS 234B $\times$ RHA 6D-1, CMS $135 \mathrm{~A} / \mathrm{CMS} 135 \mathrm{~B} \times$ GKVK-3 and NDCMS 4A/NDCMS 4B $\times$ RHA 6D-1 were in favour of male sterile cytoplasm and four viz., CMS
17A/CMS 17B $\times$ RHA 6D-1, CMS 17A/CMS 17B $\times$ RHA 23, CMS 135A/CMS 135B $\times$ RHA 93 and CMS 135A/CMS 135B $\times$ RHA 23 were in favour of male fertile cytoplasm. The $\mathrm{A} \times \mathrm{R}$ group of hybrids and their corresponding $\mathrm{B} \times \mathrm{R}$ group of hybrids did not show significant differences for seed yield plant $^{-1}$ which is evident from non-significant 
difference between overall means of $\mathrm{A} \times \mathrm{R}$ and $\mathrm{B} \times \mathrm{R}$ hybrids.

\section{Hull content}

Hull content ranged from $28.32 \%$ (CMS335A $\times$ RHA 95-C-1) to $41.42 \%(\mathrm{CMS} 135 \mathrm{~A} \times$ RHA 95-C-1) among the $\mathrm{A} \times \mathrm{R}$ hybrids and it ranged from $28.01 \%$ (CMS 234B $\times$ RHA 23) to $44.53 \%$ (CMS 17B $\times$ RHA 95-C-1) among $\mathrm{B} \times \mathrm{R}$ hybrids. $\mathrm{A} \times \mathrm{R}$ and $\mathrm{B} \times \mathrm{R}$ hybrids differed significantly in their per se performance for hull content in nine of the 25 nuclear genetic backgrounds. The $\mathrm{A} \times \mathrm{R}$ group of hybrids and their corresponding $\mathrm{B} \times$ $\mathrm{R}$ group of hybrids showed significant differences for hull content which is evident from significant difference between overall means of $\mathrm{A} \times \mathrm{R}$ and $\mathrm{B} \times \mathrm{R}$ hybrids. So, $\mathrm{B} \times \mathrm{R}$ group of hybrids were found to be superior to $\mathrm{A} \times \mathrm{R}$ group of hybrids with respect to hull content.

\section{Oil content}

The $\mathrm{A} \times \mathrm{R}$ hybrids varied for oil content from $33.15 \%(\mathrm{CMS} 335 \mathrm{~A} \times \mathrm{RHA} 93)$ to $38.94 \%$ $(\mathrm{CMS}$ 234A $\times$ RHA 6D-1) while it ranged from $33.63 \%(\mathrm{CMS} 17 \mathrm{~B} \times \mathrm{RHA} 23)$ to $39.13 \%$ (NDCMS 4B $\times$ GKVK 3) among the $\mathrm{B} \times$ Rhybrids. $\mathrm{A} \times \mathrm{R}$ and $\mathrm{B} \times \mathrm{R}$ hybrids differed significantly with respect to their per se performance only in five nuclear genetic backgrounds. The $A \times R$ group of hybrids and their corresponding $\mathrm{B} \times \mathrm{R}$ group of hybrids manifested significant differences for oil content which is evident from significant difference between overall means of $\mathrm{A} \times \mathrm{R}$ and $\mathrm{B} \times \mathrm{R}$ hybrids. Further, $\mathrm{B} \times \mathrm{R}$ group of hybrids showed superiority over $A \times R$ group of hybrids.

\section{Days to maturity}

The hybrids varied for days to maturity with a narrow range. Accordingly, it ranged from 90 days (CMS 234A × RHA 93 and NDCMS 4A $\times$ RHA 23) to 97 days (CMS 335A based hybrids) among the $\mathrm{A} \times \mathrm{R}$ hybrids while it ranged from 90 days (CMS 135B $\times$ RHA 6D1) to 98 days (CMS $335 \mathrm{~B} \times \mathrm{GKVK} 3$ ) among $\mathrm{B} \times \mathrm{R}$ hybrids. Only two of the 25 nuclear genetic backgrounds manifested significant differences between $\mathrm{A} \times \mathrm{R}$ and their corresponding $\mathrm{B} \times \mathrm{R}$ hybrids with respect to per se performance for days to maturity, both being in favour of male fertile cytoplasm. The $A \times R$ group of hybrids and their corresponding $\mathrm{B} \times \mathrm{R}$ group of hybrids did not differ significantly for days to maturity which is evident from non-significant difference between overall means of $\mathrm{A} \times \mathrm{R}$ and $\mathrm{B} \times \mathrm{R}$ hybrids.

\section{Overall per se performance status}

The iso-nuclear alloplasmic hybrids differed for their overall per se performance in eight nuclear genetic backgrounds (Table 4). Among them, $\mathrm{B} \times \mathrm{R}$ hybrids performed better than the $A \times R$ hybrids in five nuclear genetic backgrounds viz., CMS335 A/B × RHA 23, CMS 234 A/B $\times$ RHA 6D-1, CMS 234 A/B $\times$ RHA 93, NDCMS4 A/B $\times$ RHA 6D-1 and NDCMS4 A/B $\times$ RHA 95-C-1. The A $\times \mathrm{R}$ hybrids performed better in three nuclear genetic backgrounds viz., CMS335 A/B × GKVK-3, CMS 17 A/B $\times$ RHA 23 and CMS $135 \mathrm{~A} / \mathrm{B} \times \mathrm{RHA} 23$ across all the traits.

The A lines as well as B lines showed significant combining ability effects across all the traits except for hull content indicating that they differed among themselves in their abilities to transmit genes having additive effect with testers. Also, the A and B lines (in case of five traits) differed for their overall ability in combining with common testers used in the study. Similar results were reported by Neelavva (2012) in chilli. The iso-nuclear alloplasmic lines (A \& B lines) were found to interact with the common 
testers for plant height, seed yield plant-1 and hull content. Similar findings were also reported by Ramesh et al., (2006) in sorghum, Chandrashekar et al., (2007) in pearl millet and Neelavva (2012) in chilli. Further, the male sterile lines showed interaction with common testers for head diameter, stem diameter and oil content. These results provided statistical validity to compare and contrast $\mathrm{A} \times \mathrm{R}$ and $\mathrm{B} \times \mathrm{R}$ hybrids for their per se performance. The cytoplasm showed significant influence on the performance of hybrids for hull content and oil content which was evident from significant differences between overall means. The $\mathrm{A} \times \mathrm{R}$ group of hybrids showed superiority over $\mathrm{B} \times \mathrm{R}$ hybrids for hull content. The $\mathrm{B} \times \mathrm{R}$ hybrids were superior to $\mathrm{A} \times \mathrm{R}$ hybrids for oil content.

The non-significant differences between $\mathrm{A} \times$ $\mathrm{R}$ and $\mathrm{B} \times \mathrm{R}$ hybrids across all nuclear genetic backgrounds depicted that the cytoplasm did not have significant influence on per se performance for days to 50 per cent flowering. Similar results were reported by Moran et al., (2003) in sorghum, Reddy et al., (2006) in sorghum, Rosamma et al., (2007) in rice and Reddy et al., (2007) in sorghum. A × $\mathrm{R}$ and $\mathrm{B} \times \mathrm{R}$ hybrids did differ significantly in few nuclear genetic backgrounds for remaining traits i.e., head diameter, stem diameter, volume weight, 100 seed weight, seed yield plant ${ }^{-1}$, hull content, oil content and days to maturity. However, these differences were observed in both positive and negative directions with varying magnitudes dependent on nuclear background. The results were in agreement with the results reported by Hariprasanna et al., (2006) in rice, Faiz et al., (2007) in rice, Haritha (2011) in chilli and Neelavva (2012) in chilli.

Overall per se performance status of the $\mathrm{A} \times$ $\mathrm{R}$ and corresponding $\mathrm{B} \times \mathrm{R}$ hybridsprovide weak evidence for the presence of effect of male sterility inducing cytoplasm on the per se performance of hybrids. Though there were significant differences in few nuclear genetic backgrounds, they did not show any trend in favour of a particular cytoplasm. For example, $\mathrm{B} \times \mathrm{R}$ hybrids performed better than $\mathrm{A} \times \mathrm{R}$ hybrids in five nuclear genetic backgrounds while the reverse was true in three nuclear genetic backgrounds. Similar results were reported by Nanda (2011), Haritha (2011) and Neelavva (2012) in chilli.

The significant variation observed within the $A \times R$ group of hybrids and $B \times R$ group of hybrids provided statistical basis for comparing the $\mathrm{A} \times \mathrm{R}$ and $\mathrm{B} \times \mathrm{R}$ hybrids to assess the effect of cytoplasm. A $\times \mathrm{R}$ hybrids as a group differed significantly from the group of $\mathrm{B} \times \mathrm{R}$ hybrids only for hull content, oil content and oil yield. This also supported the idea of comparing $\mathrm{A} \times \mathrm{R}$ and $\mathrm{B} \times \mathrm{R}$ hybrids to assess the effect of cytoplasm for per se performance. Influence of male sterility inducing cytoplasm was evident in the expression of hybrids mean performance for few traits which was indicated from significant difference between $A \times R$ and $B \times R$ hybrids for these parameters. Such differences so obtained between isonuclear alloplasmic hybrids may not be due to the influence of cytoplasm alone, but also due to its interaction with testers.

However, it is difficult to discern the effects of the cytoplasm per se and cytoplasm $\times$ nuclear interactions. The present results provided no reasons to believe the presence of influence of male sterility inducing cytoplasm effects on the expression of hybrids for overall per se performance. However the influence was not in favour of any particular cytoplasm. To conclude, there is no significant influence of cytoplasm on seed yield and its attributing traits of sunflower as evident from the comparison of $\mathrm{A} \times \mathrm{R}$ and their corresponding $\mathrm{B} \times \mathrm{R}$ hybrids. 


\section{References}

Aruna, C., Shotria, P. K., Pahuja, S. K., Umakanth, A. V., Venkatesh, B. B., Devender, A. V. and Patil, J. V., 2013. Fodder yield and quality in forage sorghum: scope for improvement through diverse male sterile cytoplasms. Crop Pasture Sci., 63(12): 1114-1123.

Arunachalam, V. and Bandyopadhyay, A., 1979. Are "multiple cross -multiple pollen hybrids" an answer for productive populations in Brassica campestris variety Brown sarson? PartII-Evaluation of Mucromphs. Theor. Appl. Genet., 58: 5-10.

Arunachalam, V., 1974. The fallacy behind the use of a modified line $\times$ tester design. Indian J. Genet., 34: 280-287.

Bartlett, M. S., 1937. Some examples of statistical methods of research in agriculture and applied biology. J. Roy. Stat. Soc., 4: 137-170.

Chandrashekara, A. C., Prasanna, B. M., Singh, B., Bunnikrishnan, K. V. and Seetharam, A., 2007. Effect of cytoplasm and cytoplasm nuclear interaction on combining ability and heterosis for agronomic traits in pearl millet. Euphy., 153: 15-26.

Davde, J., Ecochard, R. and Marmey, P., 1989. The possible influence of cytoplasm on the performance of reciprocal soybean hybrids. Euphy., 44(1-2):49-53.

Faiz, F. A., Ijaz, M., Awan, T. H., Manzoor, Z., Ahmad, M., Wariach, N. M. and Zahid, M. A., 2007. Effect of wild abortive cytoplasm inducing male sterility on resistance/tolerance against brown plant hopper and white backed plant hopper in Basmati Rice hybrids. $J$. Anim. Pl. Sci., 17(1-2): 16-20.

Hariprasanna, K. Zaman, F. U. and Singh, A. K., 2006. Influence of male sterile cytoplasms on the physico-chemical grain quality traits in hybrid rice (Oryza sativa L.). Euphy., 149: 273-280.

Haritha, B., 2011. Effect of cytoplasm on fruit yield and its attributes and response to anthracnose resistance in chilli. (Capsicum annuum L.). M.Sc. (Agri) Thesis, Univ. Agric. Sci., Bangalore.

Kadoo, N. Y., Zaman, F. U., Singh, A. K. and Desmukh, P. S., 2002. Effects of male sterility inducing cytoplasm on morphophysiological and biochemical characters in rice (Oryza sativa L.). Indian J. Genet. Plant Breed., 62(4): 189-195.

Kempthorne, O., 1957. An introduction to genetic statistics. John Wiley and Sons, New York, 408-711.

Kumar, R. and Sagar, P., 2009. Effect of cytoplasm on downy mildew vulnerability in pearl millet (Pennisetum glaucum L. R. Br).Indian J. Genet., 69(2):115-121.

Kumar, R. and Sagar, P., 2010. Effect of cytoplasm on combining ability and yield attributes in pearl millet (Pennisetum glaucum L. R. Br).Indian J. Genet., 70(3): 247-256.

Kumar, R. and Sagar, P., 2010. Effect of cytoplasm on combining ability and yield attributes in pearl millet (Pennisetum glaucum L. R. Br).Indian J. Genet., 70(3): 247-256.

Liu, Z. O. and Li, Y. C., 1994, Heterosis of grain weight in wheat hybrid with Triticum timopheevi cytoplasm. Euphy., 75 (3): 189-193.

Mohan Rao, A., 2001. Heterosis as a function of genetic divergence in sunflower (Helianthus annuus L.). Ph.D thesis, Acharya N. G. Ranga Agril. Univ.. Hyderabad.

Moran, J. L. and Rooney, W. L., 2003. Effect of cytoplasm on the agronomic performance of grain sorghum hybrids. Crop Sci., 43: 777-781.

Mosjidis, J. A. and Yermanos, D. M., 1984. 
Maternal effects and cytoplasmic inheritance of oleic and linoleic acid contents in sesame. Euphy. 33(2): 427432.

Nanda, C., 2005. Studies on the effect of cytoplasm on combining ability and heterosis for important agronomic traits in chilli (Capsicum annuum L.). M.Sc. (Agri) Thesis, Univ. Agric. Sci., Bangalore.

Neelavva, K., 2012. Does cytoplasm influence the expression of fruit yield, fruit quality traits and responses to anthracnose disease and thrips infestation in chilli (Capsicum annuum L.)? M.Sc. (Agri) Thesis, Univ. Agril. Sci., Bangalore.

Ramesh, S., Reddy, B. V. S., Sanjana, P. R. and Ramaiah, B., 2006. Influence of Cytoplasmic-nuclear male sterility on agronomic performance of sorghum hybrids. Int. Sorghum and Millets Newsl, 47: 21-25.

Reddy B. V. S., Ramesh, S., Sanjana, P. R. and Kumar, A. A., 2009. Male-sterility inducing cytoplasmic effects on combining ability in sorghum [Sorghum bicolor (L.) Moench]. Indian J. Genet., 69(3): 199-204.

Reddy, B. V. S., Ramesh, S., Sanjana, P. R. and Ramaiah, B., 2007. Combining ability and heterosis as influenced by male-sterility inducing cytoplasms in sorghum [Sorghum bicolor (L.) Moench]. Euphy., 154: 153-164.

Rosamma, C. A. and Vijayakumar, N. K., 2007. Variation in quantitative characters and heterosis in $\mathrm{F}_{1}$ rice (Oryza sativa L.) hybrids as affected by male sterile cytoplasm. Indian J. Genet., 67(1): 142-144.

Sanjana, R. P., Rao, M. D., Reddy, B. V. S., Kumar, A. A., Thakur, R. P. and Rao, V. P., 2011. Evaluation of $A_{1}, A_{2}, A_{3}$, $\mathrm{A}_{4(\mathrm{M})}, \mathrm{A}_{4(\mathrm{G})}$ and $\mathrm{A}_{4(\mathrm{VZM})}$ cytoplasms in isonuclear backgrounds for grain mold resistance in sorghum [Sorghum bicolor (L.) Moench]. Crop prot., 30: 658-662.

Serieys, H., Griveau, Y., Kaan, F. and Berville, A., 1992. Reciprocal cross and cytoplasmic effects on agronomic traits measured on alloplasmic hybrids of sunflower (Helianthus annuus L.). Proc. of the $13^{\text {th }}$ Inter. Sunflower Conf., 5pp.Int. Sorghum and Millets Newsl., 47: 16-20.

Thakur, R. P., Rao, V. P. and King, S. B., 1991. Influence of flowering event factors in cytoplasmic male sterile lines and $\mathrm{F}_{1}$ hybrids on infection by Claviceps fusiformis in pearl millet (Pennisetum typhoides L). Plant. Dis., 75(12): 1217-1222.

Yadav, O. P., 1999. Heterosis and combining ability in relation to cytoplasmic diversity in pearl millet (Pennisetum glaucum (L) R.B.). Indian J. Genet., 59(4): 445-450.

Yadav, O. P., 1999. Heterosis and combining ability in relation to cytoplasmic diversity in pearl millet (Pennisetum glaucum (L) R.B.). Indian J. Genet., 59(4): 445-450.

Young, J. B. and Virmani, S. S., 1990. Effects of cytoplasm on heterosis and combing ability for agronomic traits in rice (Oryza sativa L.). Euphy, 48 (2): 177188.

\section{How to cite this article:}

Sunitha, N.C. and Shadakshari, Y.G. 2018. Effect of Cytoplasm on Seed Yield and Attributing Traits in Sunflower (Helianthus annuus L.). Int.J.Curr.Microbiol.App.Sci. 7(02): 106-119. doi: https://doi.org/10.20546/ijcmas.2018.702.014 